International Journal of Thermophysics, Vol. 13, No. 4, 1992

\title{
Vibrating-Wire Viscometers for Liquids at High Pressures
}

\author{
M. J. Assael, ${ }^{1}$ C. P. Oliveira, ${ }^{2}$ M. Papadaki, ${ }^{2}$ and W. A. Wakeham ${ }^{2}$
}

Received November 19, 1991

\begin{abstract}
The design and operation of two independent vibrating-wire viscometers are described. The instruments are intended for operation in the liquid phase at pressures up to $300 \mathrm{MPa}$ and have been designed specifically for this purpose using the detailed theory of the device. Extensive evidence is adduced to demonstrate that the operation of the viscometers is consistent with the theory. Although the instruments attain a precision in viscosity measurements of $\pm 0.1 \%$, when used in an absolute mode the accuracy that can be achieved is no better than $\pm 3 \%$. However, if the instrument is calibrated for two welldefined instrumental parameters, the uncertainty in the reported viscosity is improved to $\pm 0.5 \%$. The results of measurements of the viscosity of normal heptane in the temperature range 303 to $348 \mathrm{~K}$ at pressures up to $250 \mathrm{MPa}$ made with one of the viscometers are reported. The results are shown to be totally consistent with measurements reported earlier using the instrument designed for lower pressures.
\end{abstract}

KEY WORDS: high pressure; vibrating wire; viscosity.

\section{INTRODUCTION}

The vibrating-wire viscometer has a 30-year history of application to the measurement of the viscosity of Newtonian fluids [1]. During that time the method has been refined steadily with respect to both its theory and its practical realization. However, in the last 4 years advances in several distinct areas of technology have made possible rather more rapid development of the technique. The result has been the independent construction of two new vibrating-wire viscometers, which have been described in outline

\footnotetext{
${ }^{1}$ Department of Chemical Engineering, Aristotle University, GR 54006 Thessaloniki, Greece.

${ }^{2}$ Department of Chemical Engineering and Chemical Technology, Imperial College, London SW7 2BY, United Kingdom.
} 
elsewhere [2], and reports of measurements of the viscosity of a few liquid hydrocarbons and their mixtures over a modest range of temperatures and pressures $[3,4]$. The compact nature of the viscometer and its ability to withstand application at both low and high temperatures $[5,6]$ and near the critical point of fluids [7] mean that the potential applications of the new type of instrument are numerous. For this reason the present paper is intended to provide a full description of the instruments so as to enable those interested to exploit this potential.

Evidence is adduced in the paper to support the claim that the precision of the present instruments is $\pm 0.1 \%$ in the measurement of viscosity. When used in a relative manner and calibrated with respect to viscosity data with an accuracy of $\pm 0.3 \%$, it is possible to achieve an accuracy of $\pm 0.5 \%$ in the reported viscosity. The accuracy is therefore limited in this relative mode by the lack of suitable viscosity data for calibration. When operated in an absolute manner, the viscometer has an accuracy of only $\pm 3 \%$, which is then limited by the uncertainty with which it is possible to determine the radius and density of the vibrating wire.

\section{THEORY OF THE METHOD}

A complete reworking of the theory of the vibrating-wire viscometer has been provided by Retsina et al. [8] including a consideration of the conditions for its validity and all necessary corrections. Here, therefore, it is sufficient to present a summary in order to provide a background for subsequent material.

The ideal model of the viscometer consists of a circular-section beam of radius $R$, half-length $L(L \gg R)$, and density $\rho_{\mathrm{s}}$ subject to a tension $T$ and clamped at both ends. The beam is set into oscillation in a single mode within a plane containing the axis of the beam and perpendicular to it by means of an initial displacement at time $t=0$ in an initially stationary fluid of constant viscosity $\mu$ and density $\rho$.

In any one mode, $j$, the displacement $\xi_{j}$ of each point on the wire at distance $\zeta$ conforms to the equation

$$
\xi_{j}(\zeta, t)=A_{j} \phi_{j}(\zeta) e^{(i-\Delta) \omega_{j} t}+\sum_{m=1}^{\infty} g_{m} \phi_{m}(\zeta) e^{-v_{m} \omega_{m} t}
$$

in which $\phi_{j}(\zeta)$ are the eigenvectors of the normal modes of oscillation. Here the first term represents a damped single-harmonic motion, while the second summation represents an additional decaying motion owing to an initial transient in the fluid motion [8]. A complete analysis of the 
transient terms in Eq. (1) is not available. However, it has been shown [8] that for $\rho / \rho_{\mathrm{s}} \ll 1$,

$$
\begin{aligned}
& v_{\mathrm{m}} \sim 0(A) \ll 1 \\
& \frac{g_{\mathrm{m}}}{A_{\mathrm{m}}} \simeq-2 \frac{\rho}{\rho_{\mathrm{s}}} v_{\mathrm{m}} \ll 1
\end{aligned}
$$

thus the transient terms decay at the same rate as the principal oscillations but have a small amplitude. In the other extreme [8], $\rho / \rho_{\mathrm{s}} \simeq 0(1)$,

$$
v_{\mathrm{m}} \gg \Delta
$$

and

$$
g_{\mathrm{m}} \sim 0(1)
$$

so that the transient terms have a large initial amplitude but decay very rapidly. Thus, in either of these extreme cases, it is anticipated that after the first few oscillations the second term in Eq. (1) is negligible. While it may be inferred from this result that the same pertains for intermediate cases, it has not been proved so that it will always be important to demonstrate that the motion of the wire conforms to the damped, simple harmonic motion of the first term of Eq. (1), which implies that observations of the details of the motion must be possible.

The relationship between the frequency $\omega$ and the decrement $\Delta$ of the oscillation of the wire in a fluid to the properties of the fluid is made possible by a solution of the Navier-Stokes equations. Such a solution has been obtained for an infinite volume of fluid in which the effects of compressibility and the nonlinear inertial terms are negligible. The first condition implies that the circumstances should be such that the Mach number

$$
\mathrm{Ma}=\left(1+\Delta^{2}\right)^{1 / 2} \omega \varepsilon R / c \ll 1
$$

and the second that

$$
\varepsilon \ll \rho \omega R^{2} / \mu=\Omega \ll 1 / \varepsilon^{2}
$$

Here $\varepsilon=\xi_{\max } / R$ is the maximum amplitude of the motion, expressed in terms of the beam radius, and $c$ is the sonic velocity in the fluid. For a particular fluid, or range of fluids, conditions expressed by Eqs. (4) and (5) evidently dictate the frequency of operation, the radius of the wire to be employed, and the amplitude of motion to be used. 
According to Retsina et al. [8] the characteristics of the oscillation in the fluid are related to the properties of the fluid by the equations

$$
\Delta=\frac{\left(\rho / \rho_{\mathrm{s}}\right) k^{\prime}+2 \Delta_{0}}{2\left[1+\left(\rho / \rho_{\mathrm{s}}\right) k\right]}
$$

and

$$
\frac{\omega^{2}}{\omega_{0}^{2}}\left\{\left[1+\left(\rho / \rho_{\mathrm{s}}\right) k\right]\left[\Delta^{2}-1\right]-\Delta\left[\left(\rho / \rho_{\mathrm{s}}\right) k^{\prime}+2 \Delta_{0}\right]\right\}+1+\Delta_{0}^{2}=0
$$

in which $\omega_{0}$ and $\Delta_{0}$ are, respectively, the frequency and decrement of the oscillation in vacuo.

Here the quantities $k$ and $k^{\prime}$ are related to $\Omega$, where $\varepsilon \Omega$ is the Reynolds number for the flow, by the equations

$$
k=-1+2 \operatorname{Im}(A)
$$

and

$$
k^{\prime}=2 \operatorname{Re}(A)+2 \operatorname{Im}(A)
$$

where

$$
A=(i-\Delta)\left\{1+\frac{2 K_{1}(s)}{s K_{0}(s)}\right\}
$$

and

$$
s=[(i-\Delta) \Omega]^{1 / 2}
$$

The symbols $K_{0}$ and $K_{1}$ represent modified Bessel functions of zeroth and first order.

Either Eq. (6) or Eq. (7), together with Eqs. (8) to (11), provides the means to evaluate the viscosity of the fluid from measurements of $\Delta, \omega$, and $\Delta_{0}$ and a knowledge of $\rho_{\mathrm{s}}$ and $R$ and the density of the fluid $\rho$. Equations (6) and (7) are not equivalent because they differ in their sensitivity to the viscosity [8]. Equation (6) is always to be preferred in practice [8].

As a consequence, Eqs. (6) and (8) to (11) constitute the basic working equations of the experimental method provided that the conditions attached to the validity are satisfied. These conditions, themselves, therefore form constraints on the design of the instrument in a manner to be described in the next section. It may also be noted here that various other forms of working equation have been proposed involving analytic 
representations of the various Bessel functions in certain ranges [1]. Given the availability of an efficient numerical algorithm for the evaluation of the complete functions [9], such approximations are now unnecessary.

\subsection{Corrections}

There are inevitably some small corrections to the ideal model that have to be considered in applying the theory given above to any practical instrument. Many of them have been considered by Retsina et al. [8] including irreversible viscous heat generation, nonuniformities in the cross section of the beam, and the presence of a bounding surface containing the test fluid. It is also necessary to consider the effects of the failure to constrain the vibrations to a single plane.

Of the effects considered by Retsina et al. [8], the only one of significance (an effect greater than $0.01 \%$ ) on the evaluation of the viscosity arises from the presence of an outer boundary to the fluid. Chen et al. [10] have considered the motion of the wire in a fluid confined within a cylinder of radius $R_{\mathrm{c}}$. Their result shows that Eq. (6) still pertains for the observed decrement but that now

$$
\begin{aligned}
k & =\operatorname{Re}(\tilde{H}) \\
k^{\prime} & =-\operatorname{Im}(\tilde{H})+\Delta[1+\operatorname{Re}(\tilde{H})]
\end{aligned}
$$

where

$$
\widetilde{H}=H(1+i \Delta)-1
$$

and

$$
\begin{aligned}
H=\{ & 2 h^{2}\left[I_{0}(h) K_{0}\left(h^{*}\right)-I_{0}\left(h^{*}\right) K_{0}(h)\right]-4 h\left[I_{1}(h) K_{0}\left(h^{*}\right)-I_{0}\left(h^{*}\right) K_{1}(h)\right] \\
& \left.+\frac{4 h}{\sigma^{*}}\left[I_{0}(h) K_{1}\left(h^{*}\right)+I_{1}\left(h^{*}\right) K_{0}(h)\right]-\frac{8}{\sigma^{*}}\left[I_{1}(h) K_{1}\left(h^{*}\right)-I_{1}\left(h^{*}\right) K_{1}(h)\right]\right\} \mid \\
& \left\{\frac { h ^ { 2 } } { \sigma ^ { * 2 } } ( \sigma ^ { * 2 } - 1 ) \left[I_{0}(h) K_{0}\left(h^{*}\right)-I_{0}\left(h^{*}\right) K_{0}(h)\right.\right. \\
& +\frac{2 h}{\sigma^{*}}\left[I_{0}(h) K_{1}\left(h^{*}\right)-I_{1}\left(h^{*}\right) K_{0}\left(h^{*}\right)+I_{1}\left(h^{*}\right) K_{0}(h)-I_{0}\left(h^{*}\right) K\left(h^{*}\right)\right] \\
& \left.+\frac{2 h}{\sigma^{* 2}}\left[I_{0}\left(h^{*}\right) K_{1}(h)-I_{0}(h) K_{1}(h)+I_{1}(h) K_{0}\left(h^{*}\right)-I_{1}(h) K_{0}(h)\right]\right\}
\end{aligned}
$$


with

$$
\begin{gathered}
h=\{(1+i \Delta) i \Omega\}^{1 / 2} \\
h^{*}=\{(1+\Delta) i \Omega\}^{1 / 2} \sigma^{*}
\end{gathered}
$$

and

$$
\sigma^{*}=\frac{R_{\mathrm{c}}}{R}
$$

In these equations $I_{0,1}$ and $K_{0,1}$ are modified Bessel functions. Although Eqs. (12) to (18) could be incorporated into the working equations, it is preferable to proceed in a different way because it is impossible in practice to arrange an entirely circular cross section for the solid materials surrounding the wire. Accordingly, for a particular geometric arrangement, we define $\mu$ as the solution for the viscosity of a fluid obtained from Eqs. (6) and (8)-(11) for given measurements of $\Delta, \omega$, and $\Delta_{0}$, and $\mu^{\prime}$ as the solution for the viscosity of the same fluid obtained from Eqs. (6) and (12)-(18) using the same values of $\Delta, \omega$, and $\Delta_{0}$. For a finite value of $\sigma^{*}$ in the instrument $\mu$ and $\mu^{\prime}$ will differ, with $\mu^{\prime}$ being the correct viscosity. Thus, if measurements are interpreted with Eqs. (6) and (8)-(11) it is possible to define a correction

$$
\delta \mu=\mu-\mu^{\prime}
$$

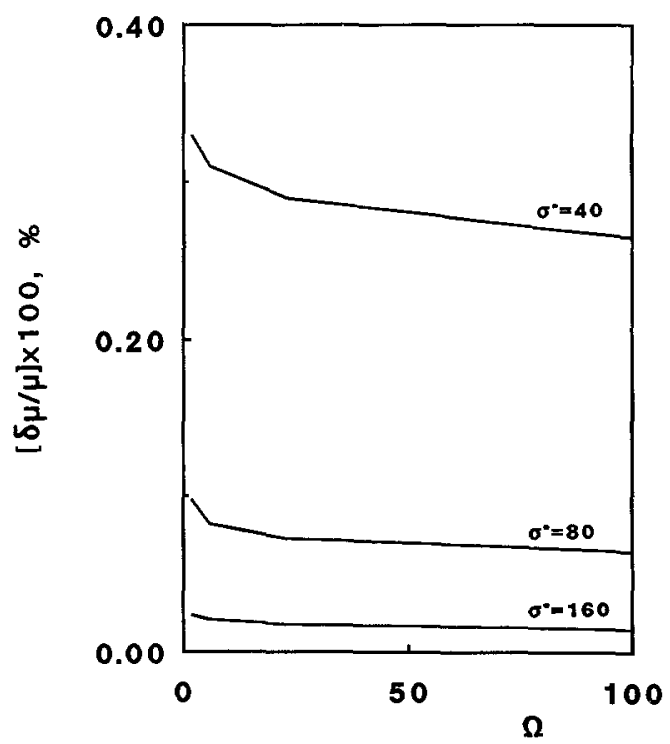

Fig. 1. The fractional correction $\delta \mu$ arising from the effect of an outer boundary as a function of $\sigma^{*}$ and $\Omega$. 
which is to be added to the value of $\mu$ to yield a corrected viscosity. It follows that one purpose of the design of the viscometer could be to reduce $\delta \mu$ or, more properly, $\delta \mu / \mu$, to a sufficiently small value that the correction is negligible. For the case when the outer surface of the fluid is not cylindrical, such a course of action is, of course, essential, and in such a calculation, the value of $R_{\mathrm{c}}$ should be taken as the distance between the wire and the nearest bounding surface.

Figure 1 shows the relative error $\delta \mu / \mu$ as a function of $\sigma^{*}$ and $\Omega$. It can be seen that provided $\Omega$ lies in the range $1 \leqslant \Omega \leqslant 90$ and $\sigma^{*}>50$, the correction to the viscosity does not exceed $0.2 \%$. For $\sigma^{*} \geqslant 80$ the correction is sufficiently small $(0.1 \%)$ that, even if its evaluation is approximate, because of a failure of the practical design to match exactly the mathematical model, the consequences for the application of the correction expressed by Eq. (19) introduce a negligible additional error into the reported viscosity.

\section{INSTRUMENT DESIGN}

\subsection{Design Constraints}

Naturally, in order that Eqs. (6) and (8)-(11) can be used to interpret experimental data, it is necessary to ensure that the instrument configuration conforms to the conditions imposed on the validity of these equations. In practice, it is preferable to express these conditions in terms of limitations on the frequency of oscillation and radius of the wire for specific choices of the physical properties of the latter. Thus, we obtained from the inequalities expressed by Eq. (5),

$$
\omega \ll \mu / \varepsilon^{2} \rho R^{2}
$$

and

$$
\omega \gg \varepsilon \mu / \rho R^{2}
$$

which provide upper and lower bounds in the space $(R, \omega)$ for specific ranges of $\mu$ and $\rho$ for the fluid as well as the obvious condition $\varepsilon \leqslant 1$. In addition, the condition given by Eq. (4) requires that

$$
\omega \ll c / \varepsilon R
$$

These conditions make it clear that the choice of the smallest value of $\varepsilon$ (the amplitude of the wire motion as a fraction of radius) which is practicable provides the best design. Accordingly, it is necessary to consider practical constraints before proceeding further. 
It is well-known from work with other oscillatory viscometers [11] that the decrement, $\Delta$, can be determined with a high precision only if its value is in the range

$$
0.005 \leqslant \Delta \leqslant 0.1
$$

This is because, for smaller values of $\Delta$ the rate of change of amplitude with time is too small to be determined precisely, whereas with bigger values insufficient oscillations can be observed. Thus, if the decrement of the oscillation of the wire was to be determined to within $\pm 0.1 \%$ from direct measurements of the displacement, it is easily shown that a spatial resolution, $\delta$, of

$$
\delta=2 \times 10^{-4} \varepsilon R
$$

has to be achieved. Since $\varepsilon \leqslant 1$, this result implies an unattainable spatial resolution $\left(10^{-10} \mathrm{~m}\right)$ for any practical value of $R$. On the other hand, if electromagnetic induction is employed to detect the velocity of the wire, then the consequent resolution in the induced voltage required to achieve the same level of accuracy in the measurement of the decrement is

$$
\delta_{\mathrm{v}}==2 \times 10^{-4} \gamma \varepsilon R \omega
$$

in which $\gamma$ is a combined transducer/amplification factor. For those values of $R$ and $\omega$ likely to be adopted in practice, and the attainable values of $\gamma$, a choice of $\varepsilon \sim 10^{-2}$ yields a required voltage resolution of $0.2 \mu \mathrm{V}$. This is comparable with the noise levels attainable with conventional circuitry and therefore provides a practical means of attaining the necessary precision in the measurement of $\Delta$.

Use of the value $\varepsilon \sim 10^{-2}$ in Eqs. (20) and (21), together with the adoption of the physical properties of the fluid that it is desired to study, defines a region in $(\omega, R)$ space in which the viscometer must be designed. The present viscometers are designed for the study of hydrocarbon liquids at pressures up to $300 \mathrm{MPa}$ for which $\mu$ and $\rho$ lie in the ranges

$$
\begin{gathered}
10^{-1} \leqslant \mu \leqslant 10 \mathrm{mPa} \cdot \mathrm{s} \\
6 \times 10^{2} \leqslant \rho \leqslant 10^{3} \mathrm{~kg} \cdot \mathrm{m}^{-3}
\end{gathered}
$$

and these values have been used in constructing the appropriate limits of operation shown in Fig. 2. The same figure includes the condition imposed by inequality (22). Furthermore, for each pair of values of $R$ and $\omega$ and a selection of wire materials $\left(\rho_{\mathrm{s}}^{\mathrm{s}}\right)$, the working equations $(6)$ and $(8)-(11)$ 


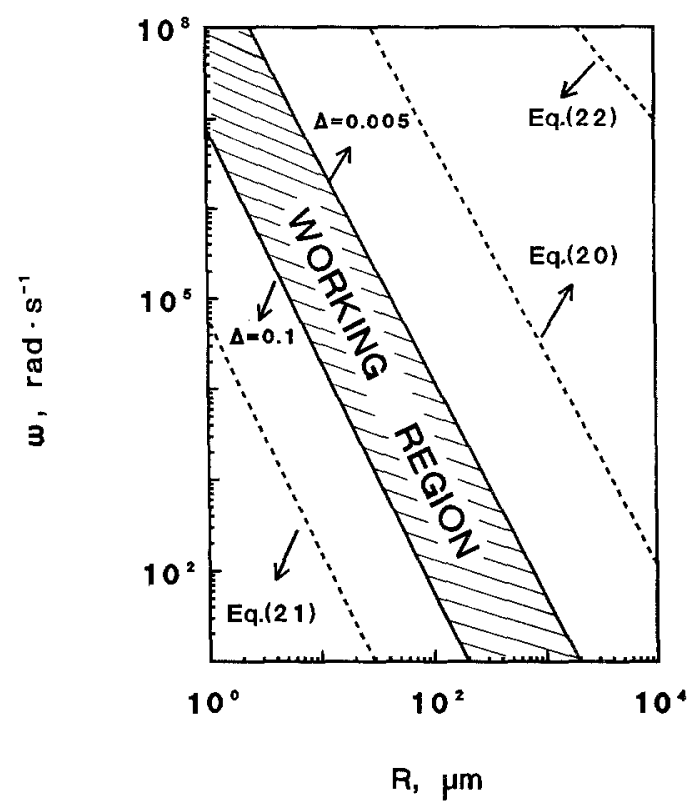

Fig. 2. The working region for the viscometer.

permit the determination of the corresponding decrement which should lie within the range defined by inequality (23). Figure 2 includes the corresponding limits for tungsten. The hatched region in Fig. 2 then defines the zone in which the viscometer must operate. For other wire materials it is quite possible that the region defined by the conditions on the decrement $\Delta$ lies outside of the region prescribed by the conditions on the validity of the working equations so that they are completely eliminated from consideration.

The final step in the design process is then the selection of the length of wire, $2 L$, and the tension, $T$, imposed upon it to achieve its desired frequency. These quantities are, of course, related by the result

$$
\omega \simeq \omega_{0} \simeq \frac{\pi}{L}\left(\frac{T}{\rho_{\mathrm{s}} \pi R^{2}}\right)^{1 / 2}
$$

This equation can be used to construct a further series of lines in the $(R, \omega)$ space for various $T, L$, and $\rho_{\mathrm{s}}$, and those combinations that fall within the working range are, in principle, acceptable configurations for the instrument. However, one final constraint must be imposed: that the stress in the wire imposed by the tension $T$, denoted by $\sigma$, should be less than the yield stress in order to preserve the integrity of the wire. Indeed, even in order 
to preserve the constancy of the physical properties of the wire, it is desirable that [12]

$$
T / \pi R^{2}<0.6 \sigma_{\mathrm{el}}
$$

When all of these constraints are added to those in Fig. 2, the design of the viscometer is almost completely determined.

An entirely different set of design constraints follows from the necessity to impose a tension, $T$, on the wire, in circumstances where the entire assembly may be subject to substantial changes in temperature and/or pressure. This is because of the dimensional changes that accompany such alteration in conditions. Thus, any attempt to tension the vibrating wire between the ends of a fixed frame necessarily leads to large and often irreproducible changes in the frequency of oscillation, as the differential length of the wire and its support are altered by temperature and pressure changes.

For this reason, it is desirable to seek a wire-tensioning method essentially independent of imposed temperature and pressure changes. The particular method adopted in the present viscometers is described below. The benefits and potential disadvantages of the scheme have been discussed in detail elsewhere [2] and so are not repeated here.

\section{THE VISCOMETERS}

\subsection{Construction}

Two viscometers have been designed in accordance with the principles set out above. Since the two instruments have many features in common, only one is described in detail and a schematic diagram of it is shown in Fig. 3. This instrument was designed for operation at pressures up to $300 \mathrm{MPa}$ and temperatures in the range $270-370 \mathrm{~K}$. The other instrument covers the same temperature range but has a lower pressure limit of $80 \mathrm{MPa}$.

In the instrument shown in Fig. 3, the vibrating wire is made of $100-\mu \mathrm{m}$-diameter tungsten wire of 56-mm length with a resonant frequency in vacuo of about $1 \mathrm{kHz}$. The wire is supported between two chucks. The upper chuck (2) is supported by a plate (1) and insulated from it by a PTFE washer and a ceramic cone. The support plate is itself connected to the upper closure of the high-pressure vessel (not shown). The chuck (7) at the lower end of the wire carries a central weight (9), also made of tungsten, that provides the main tension in the wire. A second weight (8), made of stainless steel, is connected to the central weight via a balance 


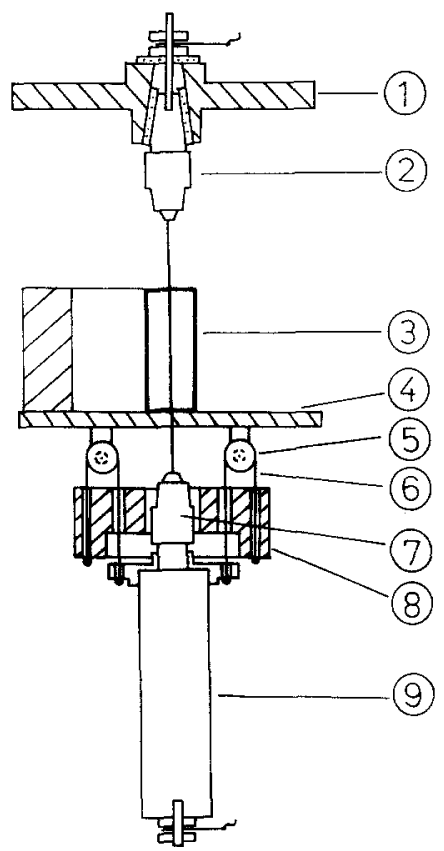

Fig. 3. Schematic diagram of the highpressure vibrating-wire viscometer.

mechanism. A tungsten wire (6) passes through a small hole in the top of the central weight and is held in place by a crimped tube. The wire then passes over a pulley (5), pivoted on two cones running in two jeweled bearings, set in the middle plate (4) and it is attached to the outer weight (8) by a mechanism similar to that used on the central weight. A suitable choice of the volumes and masses of the two weights ensures that any buoyancy force exerted on the inner weight (9), which would otherwise alter the resonant frequency of the wire in response to the fluid density changes, is compensated by an essentially equal and opposite force on the outer weight (8). At the same time the inertia of the suspended weights is such that the lower point of the attachment to the vibrating wire remains a node.

An essential feature of the compensating mechanism is that no additional frictional force should be introduced. In order to confirm that the pulley bearings provide negligible friction, a test piece was manufactured and the frictional force was determined. It was found that the equivalent mass contributed by the bearing was about $120 \mathrm{mg}$, which is negligible compared with the total effective mass of $300 \mathrm{~g}$ tensioning the wire. The 
operation of the compensation mechanism was further confirmed by the observation that the resonant frequency of oscillation of the wire changed by $2 \mathrm{~Hz}$ when the density of the fluid surrounding the weights was changed by $600 \mathrm{~kg} \cdot \mathrm{m}^{-3}$.

Electrical connections to the wire are made through the upper chuck and the central weight and are led out of the pressure vessel through appropriate feedthroughs. The oscillations of the wire are induced electromagnetically and detected in a similar way. The magnets (3), used for both purposes, are made of a samarium-cobalt alloy and produce a field of $1 \mathrm{~T}$ at the wire. The separation of the faces of the magnets was $8 \mathrm{~mm}$. They constitute the closest external boundary to the test fluid and they extend over the central $25 \mathrm{~mm}$ of the wire length. Thus, for the purposes of estimating the magnitude of the correction $\delta \mu$ discussed in Section 2.1, $R_{\mathrm{c}}$ has been taken as $4 \mathrm{~mm}$, so that $\sigma^{*} \simeq 80$. Thus, for the values of $\Omega$ employed in the present measurements the maximum corrections, $\delta \mu$, obtained by assuming a circular boundary at a distance $R_{\mathrm{c}}$ from the wire over its complete length and circumference would be $0.08 \%$. In practice, the correction will be less than this, so that if Eq. (19) is used to estimate it the error contributed to the final viscosity will be negligible.

The pressure vessel containing the viscometer is mounted vertically in an oil thermostat bath agitated by the escape of bubbles of nitrogen gas from a distribution plate mounted at the bottom of the bath. This mechanism avoided the deleterious effects of conventional mechanical stirring which introduce significant vibrations into the system. The system also secures a high degree of uniformity in the temperature (better than $\pm 10 \mathrm{mK}$ over the length of the pressure vessel). Temperature control was effected in a conventional manner and a stability of $\pm 5 \mathrm{mK}$ could be maintained for several days. The temperature was determined with a platinum resistance thermometer strapped to the pressure vessel with an accuracy of $\pm 10 \mathrm{mK}$.

The pressure vessel could be filled with the test liquid under vacuum and subsequently pressurized by an air-driven diaphragm pump. The pressure was measured with a quartz-crystal pressure transducer (Digiquartz Model $40 \mathrm{~K}-110$ ) with a resolution of $0.1 \mathrm{MPa}$. The gauge was calibrated against a deadweight tester certified by the National Physical Laboratory.

\subsection{Measurement}

A viscosity measurement consists of the initiation of a transverse vibration of the wire followed by observation of the decay of the subsequent simple harmonic motion. 
Initiation of the oscillation is achieved by applying two dc current pulses, of opposite sign to the wire, with an interval corresponding to the resonant frequency of oscillation of the wire. This action induces an oscillation which is symmetric about the rest position of the wire. The velocity of the wire's motion is then determined from the signal induced in the wire as a result of its motion in the magnetic field. The magnitude of the current pulse is adjusted in order to secure an amplitude of the wire motion no greater than $10^{-2} R$ as required by the theory.

As has been reported in detail elsewhere [2], considerable care must be taken to ensure that there is no coupling between the various modes of motion of the wire during the measurement process. In particular, the coupling of the two nearly degenerate orthogonal modes of the circularsection wire, which leads to a precession of the phase of oscillation, must be eliminated. This is because such precession reveals itself very clearly in a modulation of the observed signal induced in the wire at the frequency of the precession. It is for this reason that the buoyancy compensation mechanism contains a two-point support since it has been found that this arrangement completely eliminates coupling of the two modes and ensures oscillation of the wire in a single plane.

In order to observe the induced signal of about $360 \mu \mathrm{V}$, the vibrating wire is mounted in one arm of the bridge circuit shown in Fig. 4. The opposing arm of the bridge consists of an identical tungsten wire which is stationary. This configuration ensures that common-mode signals arising from temperature fluctuations are eliminated from the output, which is passed to a purpose-built low-impedance amplifier with a gain of $3 \times 10^{4}$. The amplified signal is then interrogated with an A/D convertor at a rate of $50 \mathrm{kHz}$ with a resolution of 1 part in $2 \times 10^{4}$, and the readings are stored

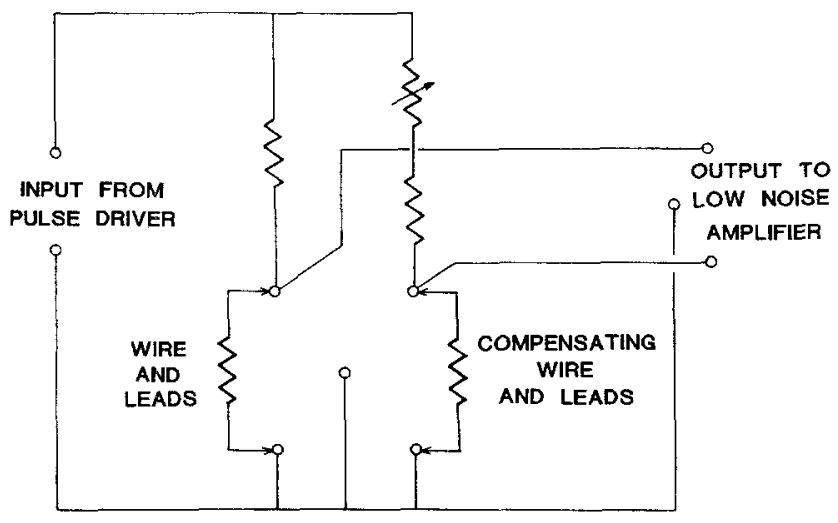

Fig. 4. Circuit diagram of the ac bridge. 
in a microcomputer via Direct Memory Access for subsequent processing. For an oscillation frequency of $1 \mathrm{kHz}$ this implies acquisition of about 50 points per cycle of the wire's motion. Observation is typically continued for $20-40$ cycles, leading to a total record of 1-2000 data during a decay of the oscillation lasting $20-40 \mathrm{~ms}$.

\subsection{Data Reduction}

Determination of the characteristics of the oscillation $\Delta$ and $\omega$ from the recorded data can be performed in a number of ways. It is possible to Fourier transform the data and to use the transform to determine the frequency, $\omega$, from the result peak in the frequency domain and the decrement, $\Delta$, from the width of the Lorentzian peak [13]. Alternatively, it is possible to determine the peak values of the velocity and use pairs of these to determine the decrement. Finally, one can simply fit the observed data to a function of the form

$$
V=A e^{-\Delta \omega t} \sin (\omega t+\phi)
$$

by the Marquard method and determine $\Delta$ and $\omega$ as coefficients of the fit. Numerical simulations of the measurement process have indicated that the final method yields the decrement with a precision of better than $\pm 0.05 \%$ and the frequency with a precision of $\pm 0.005 \%$. Furthermore, this method has the distinct advantage that it is possible unequivocally to demonstrate that the instrument operates in accordance with the theoretical model of it which predicts a motion of the form of Eq. (28). Thus, it is possible to confirm the absence of secondary wire motions including the modulation that accompanies precession of the plane of oscillation. Moreover, the same technique allows elimination from the analysis of those points acquired soon after initiation of the run, which may contain the influence of the transient terms discussed earlier as well as that of the manner of initiation. Generally, it was found necessary to eliminate the first five cycles of oscillation for this reason.

Figure 5 shows the results of a measurement in toluene at a temperature of $303 \mathrm{~K}$ and a pressure of $0.1 \mathrm{MPa}$. In Fig. 5a the solid line represents the fit of Eq. (28) to the recorded data which are shown as the points and Fig. 5b shows the corresponding deviations. The latter figure shows an essential random distribution of deviations, and taken together, the two figures confirm the correct operation of the instrument.

Repeated measurements under nominally identical conditions confirm the reproducibility and precision of the measurement of the decrement of 

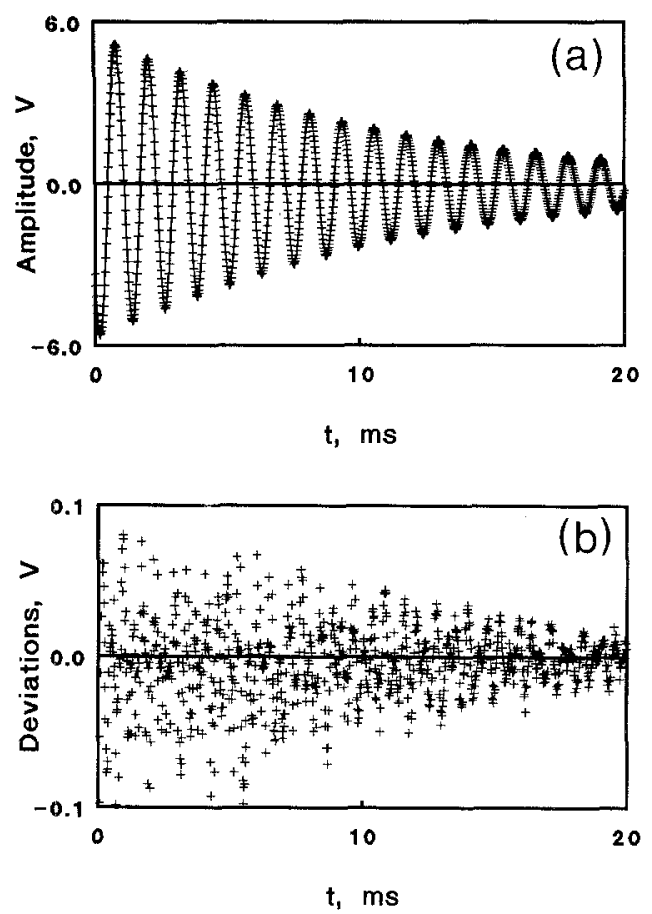

Fig. 5. (a) The transient decay of the detected signal; (b) deviations from a fit to a damped simple harmonic motion.

the oscillation $\Delta$ to be better than $\pm 0.1 \%$ and those of the frequency to be $\pm 0.01 \%$.

In order to evaluate the viscosity from Eqs. (6) and (8)-(11) it is necessary to determine $\Delta_{0}$, the decrement of the oscillation in vacuo. This measurement has been carried out in situ over a range of temperatures. The values of $\Delta_{0}$ determined are listed in Table $\mathrm{I}$. The very small magnitude of

Table I. Measurements of the

Logarithmic Decrement in Vacuo as a Function of Temperature

\begin{tabular}{cc}
\hline$T$ & \\
$(\mathrm{~K})$ & $\Delta_{0} \times 10^{5}$ \\
\hline 303 & 5.48 \\
318 & 4.06 \\
343 & 3.22 \\
\hline
\end{tabular}


$\Delta_{0}$ means that the precision of its determination is rather poor $( \pm 1 \%)$. However, because changes in $A_{0}$ of $10 \%$ affect the determination of a liquid viscosity by only $0.03 \%$, the error propagated to the reported viscosity is negligible.

\subsection{Evaluation of the Viscosity}

In addition to measurements of $\Delta$ and $\omega$ for an oscillation in the fluid and $A_{0}$, the decrement in vacuo, Eqs. (6) and (8)-(11) show that an absolute determination of the viscosity of the fluid requires values for $R$, the radius of the wire, $\rho_{\mathrm{s}}$, the density of the wire material, and $\rho$, the fluid density. The latter is usually available from independent sources with an accuracy of $\pm 0.2 \%$ or better. However, the determination of the other two quantities presents significant problems. It is not practicable to determine the diameter of a nominal $100-\mu \mathrm{m}$ wire with a precision of better than $\pm 0.1 \mu \mathrm{m}$, especially since the lack of circularity and uniformity may be of the same order. Similarly, the independent determination of the density of the wire using a sample $50-100 \mathrm{~mm}$ long with a precision of $\pm 0.1 \%$ seems to pose insuperable difficulties. Thus, the performance of absolute measurements with the viscometer, while possible, leads to results with an associated accuracy significantly inferior to the precision of the device.

For the present instruments, the diameter of the wire has been determined with an optical microscope with an error of $\pm 1 \%$. The density of tungsten available from the literature $[14,15]$ has a spread of values amounting to $\pm 0.7 \%$. Taken together, this means that the accuracy of absolute viscosity measurements is presently limited to $\pm 3 \%$.

For this reason it is preferable to use a relative method of evaluation of the viscosity in which $R$ and $\rho_{\mathrm{s}}$ are determined with respect to standard reference values for the viscosity of two or more liquid samples. The present viscometers contain elements subject to attack by water, for which the viscosity is known to within $\pm 0.1 \%$ [16]. Thus, it has been necessary to calibrate the instrument with respect to reference data for some liquid hydrocarbons.

A recent review of the viscosity of liquid normal alkanes with saturation conditions as a function of temperature conducted by Dymond and $\emptyset$ ye [17] has revealed large discrepancies among the complete set of data available. However, there are two sets of measurements where the analysis of Dymond and $\emptyset$ ye supports the estimated uncertainty of the data of $\pm 0.3 \%$. The first set comprises the measurements of the viscosity of toluene made by Gonçalves et al. [18] using a capillary viscometer, while the second set consists of the measurements reported by Knapstad et al. on a series of normal alkanes using an oscillating-cylinder viscometer [19]. 
These data therefore provide the best available body of information for the calibration of the viscometers.

Evidently, the calibration of the viscometers seeks to determine two quantities, $R$ and $\rho_{\mathrm{s}}$, so that a minimum of two calibration measurements must be performed. In fact, it is preferable to perform more calibration measurements than this as a check on the internal consistency of the calibration data. Thus, the calibration of both viscometers has been carried out with respect to five liquids at a temperature of $30^{\circ} \mathrm{C}$ under saturation conditions. The five liquids studied are toluene, $n$-hexane, $n$-heptane, $n$-octane, and $n$-decane. In order to evaluate dependent values of $R$ and $\rho_{\mathrm{s}}$ from the measurements, the results of the calibration runs with toluene and each of the normal alkanes have been paired and two simultaneous equations of the form of Eq. (6) solved to determine values of $R$ and $\rho_{\mathrm{s}}$.

Table II lists the data used for the calibration together with the individual results for $R$ and $\rho_{\mathrm{s}}$ obtained with the higher-pressure viscometer. The viscosity data have been taken from the work of Gonçalves et al. [18] for toluene and from that of Knapstad et al. [19] for the normal alkanes. The densities of the liquids have been taken from the results of Kashiwagi et al. [20] for toluene and from the work of Knapstad et al. [19] for the normal alkanes. Table II includes the results of the direct optical measurement of the radius of the wire and the value of $\rho_{\mathrm{s}}$ for tungsten obtained from the literature [14].

Two facts emerge from the table. First, the derived value of the wire radius, $R$, is consistent among the four pairs of liquids to within $\pm 0.1 \%$, while the density $\rho_{\mathrm{s}}$ has an internal consistency of $\pm 0.04 \%$. The claimed uncertainties in the calibration data indicate that the expected error in these quantities is significantly larger. This provides indirect, but strong, support for the estimated uncertainty in the viscosity data of Gonçalves et al. and Knapstad et al. The second fact of significance is that the values of $R$ and $\rho_{\mathrm{s}}$ derived by calibration are consistent with the values determined independently within the greater uncertainty of the latter. This fact

Table II. Values of Wire Radius and Wire Density Obtained by Calibration ${ }^{a}$

\begin{tabular}{lccccc}
\hline & Toluene & $n$-Hexane & $n$-Heptane & $n$-Octane & $n$-Decane \\
\hline Viscosity $\mu(\mathrm{mPa} \cdot \mathrm{s})$ & 0.52309 & 0.28096 & 0.36954 & 0.48072 & 0.79064 \\
Density $\rho\left(\mathrm{kg} \cdot \mathrm{m}^{-3}\right)$ & 857.83 & 650.41 & 675.21 & 694.73 & 722.54 \\
Wire radius $R(\mu \mathrm{m})$ & & 49.49 & 49.40 & 49.45 & 49.49 \\
Wire density $\rho_{s}\left(\mathrm{~kg} \cdot \mathrm{m}^{-3}\right)$ & & 19,231 & 19,245 & 19,239 & 19,230 \\
\hline
\end{tabular}

${ }^{a}$ Measured radius $=50.05( \pm 0.5) \mu \mathrm{m}$ Literature density $[14]=19,300( \pm 130) \mathrm{kg} \cdot \mathrm{m}^{-3}$. 
provides further confirmation that the instruments operate in accordance with the theoretical model of them and that there are no significant unaccounted systematic errors.

Calibration results for the low-pressure viscometer, which are omitted here, are consistent with those reported in Table II. For the purposes of measurements the mean value for both $R$ and $\rho_{\mathrm{s}}$ of those listed in Table II have been adopted, namely, $R=49.46 \mu \mathrm{m}$ and $\rho_{\mathrm{s}}=19,236 \mathrm{~kg} \mathrm{~m}^{-3}$. For operation over a range of temperatures and pressures it is necessary to correct the values determined at $30^{\circ} \mathrm{C}$ for thermal expansion and hydrostatic compression.

Accordingly, we have applied the equations

$$
R(t, P)=R(20,0.1)[1+\alpha(t-20)][1-(P-0.1) / 3 K]
$$

and

$$
\rho_{\mathrm{s}}(t, P)=\rho_{\mathrm{s}}(20,0.1)[1+(P-0.1) / K][1+3 \alpha(t-20)]^{-1}
$$

where $P$ is expressed in MPa and $t$ in degrees Celsius. Values of the bulk modules, $K$, and the linear coefficient of thermal expansion, $\alpha$, have been taken from the literature [15]. Even at the highest pressure and temperature, the corrections contribute less than $0.1 \%$ to the final viscosity. The additional error introduced by these corrections is negligible.

\subsection{Accuracy}

As mentioned previously, it is estimated that the precision of the measurement of the decrement of the oscillations of the wire is approximately $\pm 0.1 \%$ and that of the frequency is $\pm 0.01 \%$. The precision of the viscosity measurements is determined by these factors, together with the error in the density of the fluid. Since this is typically $\pm 0.2 \%$ the error propagated to the viscosity is of the order of $\pm 0.1 \%$. Thus, the precision of the viscosity measurements is about $\pm 0.2 \%$.

The accuracy of the reported viscosity data is, however, also influenced by the uncertainties in the values of $R$ and $\rho_{\mathrm{s}}$. In turn, these are dependent on the accuracy of the viscosity data used for calibration. As a result, it is estimated that the accuracy of the reported viscosity data is $\pm 0.5 \%$ over the entire range of temperature and pressure. The essential limitation on the accuracy therefore derives from the calibration data available and should encourage attempts to make absolute viscosity measurements of a few liquids near ambient conditions with an accuracy of $\pm 0.1 \%$ or better to serve as standard reference data. 
Table III. The Viscosity of Normal Heptane

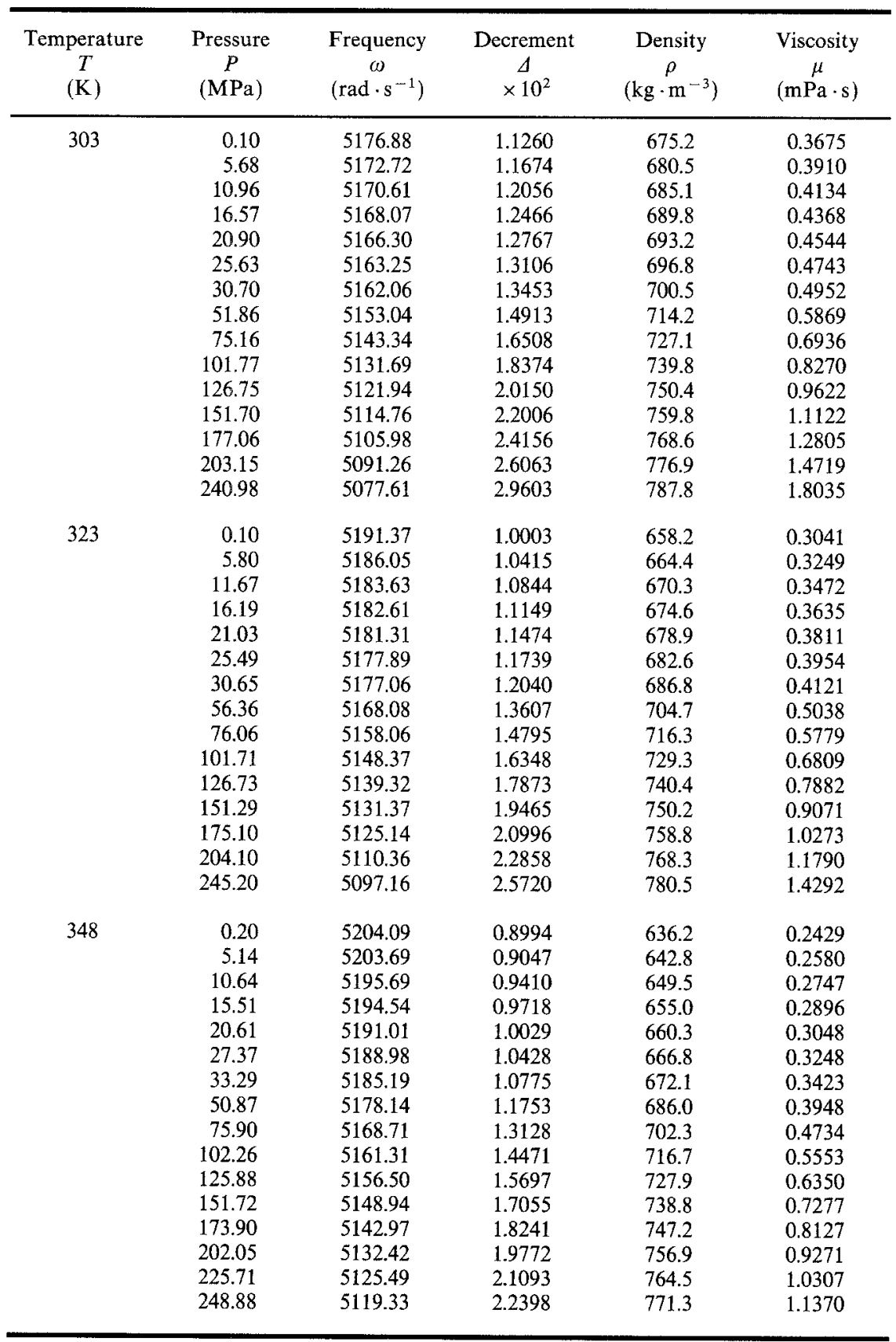




\section{RESULTS}

In the present paper we report measurements for the viscosity of $n$-heptane over a range of pressures up to $250 \mathrm{MPa}$ in the temperature range 303.15 to $348.15 \mathrm{~K}$. The measurements were carried out independently with the two viscometers. The samples of $n$-heptane were provided by Merck Ltd. with a stated purity of $99.5 \%$. The samples were further distilled and degassed before use and the purity determined chromatographically was found to be better than $99.9 \%$. The density of liquid $n$-heptane has been taken from the work of Doolittle [21] and has a reported uncertainty of $\pm 0.2 \%$.

Table III lists the results of the measurements of the viscosity of $n$-heptane determined with the high-pressure viscometer for three isotherms, whereas the values determined in the low-pressure for two isotherms have been reported earlier. The table includes the values of the measured frequency and decrement of the oscillation since these data will permit a reevaluation of the viscosity should more accurate calibration data become available in the future.

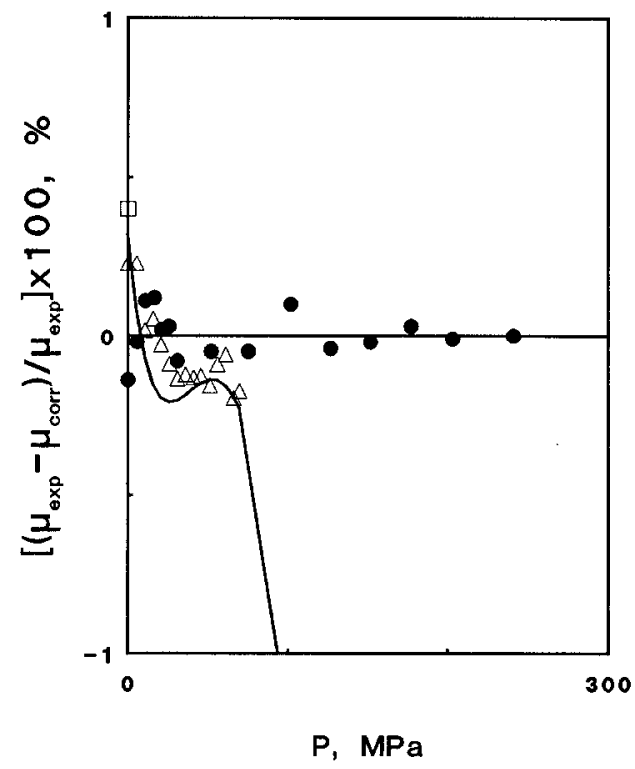

Fig. 6. Deviations of experimental viscosity data for $n$-heptane from the present representation at $303 \mathrm{~K}$. (๑) Present work; ( $\triangle$ ) Assael and Papadaki [3]; ( $\square$ ) Knapstad et al. [19]; (-) Kashiwagi and Makita [22]. 


\subsection{Comparison with Earlier Work}

Measurements of the viscosity of $n$-heptane have been carried out by Kashiwagi and Makita with a quartz-crystal viscometer [22], Assael and Papadaki [3] in the low-pressure viscometer described here, and Knapstad et al. at saturation with an oscillating-cylinder viscometer [19]. Figures 6-8 show the deviations between the various sets of results and a representation of the data obtained with the high-pressure viscometer. The representation takes the form

$$
\mu=\sum_{i=0}^{4} a_{i}\left(P / P^{\prime}\right)^{i / 2}
$$

wherein $P^{\prime}$ and the coefficients $a_{i}$ for the three isotherms are listed in Table IV. The same table lists the standard deviation of the fit of this function to the data obtained using the high-pressure viscometer.

Figures 6-8 and the values of the standard deviation confirm that the precision of the present viscosity data is of the order of $\pm 0.2 \%$. The same figures indicate that over the limited range of conditions accessible to the

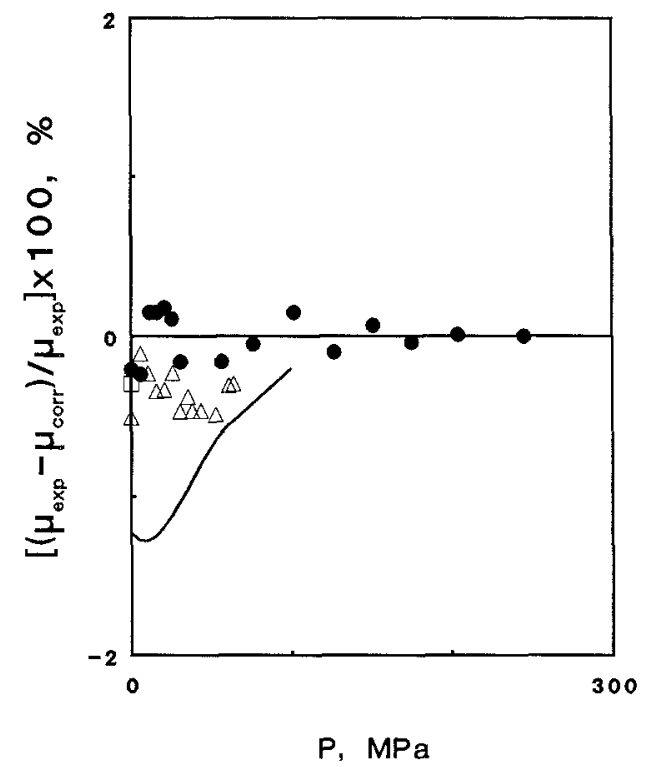

Fig. 7. Deviations of experimental viscosity data for $n$-heptane from the present representations at $323 \mathrm{~K}$. (๑) Present work; ( $\triangle$ ) Assael and Papadaki [3]; ( $\square$ ) Knapstad et al. [19]; (-) Kashiwagi and Makita [22]. 


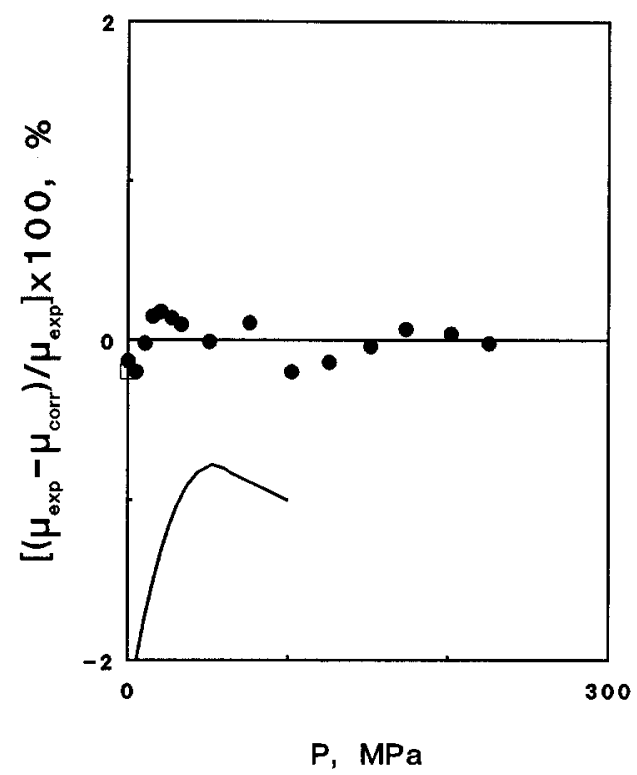

Fig. 8. Deviations of experimental viscosity data for $n$-heptane from the present representation at $348 \mathrm{~K}$. ( ) Present work; ( $\square$ ) Knapstad et al. [19]; ( ) Kashiwagi and Makita [22].

low-pressure instrument, the datá of Assael and Papadaki [3] are consistent with the present results to within $\pm 0.3 \%$, which is well within the mutual uncertainty limit of $\pm 0.5 \%$. In addition, the data of Knapstad et al. [19] along the saturation line deviated by no more than $\pm 0.5 \%$ for the present representation at any temperature, which is again consistent with the combined uncertainty. The results of Kashiwagi and Makita [22], which have a claimed uncertainty of $\pm 2 \%$ and extend up to a pressure of $100 \mathrm{MPa}$, show a rather greater and systematic deviation from the present work but it remains within the mutual uncertainty.

Table IV. Coefficients of Eq. (31)

\begin{tabular}{lccc}
\hline Coefficient & $T=303.15 \mathrm{~K}$ & $T=323.15 \mathrm{~K}$ & $T=348.15 \mathrm{~K}$ \\
\hline$a_{0}(\mathrm{mPa} \cdot \mathrm{s})$ & 0.3628 & 0.2960 & 0.2412 \\
$a_{2}(\mathrm{mPa} \cdot \mathrm{s})$ & 0.6709 & 0.0777 & 0.3994 \\
$a_{3}(\mathrm{mPa} \cdot \mathrm{s})$ & -0.4551 & 0.2842 & -0.1616 \\
$a_{4}(\mathrm{mPa} \cdot \mathrm{s})$ & 0.3455 & 0.1134 & 0.1120 \\
Standard deviation, \% & 0.20 & 0.15 & 0.10 \\
$P^{\prime}(\mathrm{MPa})$ & 120.5 & 122.7 & 124.5 \\
\hline
\end{tabular}




\section{CONCLUSIONS}

Two vibrating-wire viscometers have been constructed and operated in accordance with design specifications defined by the theory of the instrument. Measurements have been performed to confirm that the instruments conform to the theoretical description of them. It has been shown that a precision on viscosity measurements on liquids of better than $\pm 0.2 \%$ is possible over a wide range of pressures. At present the accuracy of the results is limited by the availability of standard reference data for calibration but there remains the scope for improvement. The configuration of the instruments is such that they have the potential for application over an even wider range of thermodynamic states.

\section{ACKNOWLEDGMENTS}

Financial support was provided by the U.K. National Engineering Laboratory and the British Council in Thessaloniki, Greece.

\section{REFERENCES}

1. W. A. Wakeham, A. Nagashima, and J. V. Sengers (eds.), Experimental Thermodynamics, Vol. III. Measurements of the Transport Properties of Fluids (Blackwell Scientific, Oxford, 1991).

2. M. J. Assael and W. A. Wakeham, 11th Symposium on Thermophysical Properties, Boulder, Colorado, June 1991.

3. M. J. Assael and M. Papadaki, Int. J. Thermophys. 12:801 (1991).

4. M. J. Assael, L. Karagiannidis, and M. Papadaki, Int. J. Thermophys. 12:5 (1991).

5. J. T. Tough, W. D. McCarmick, and J. G. Dash, Rev. Sci. Instrum. 35:1345 (1964).

6. J. T. R. Watson, NEL Report No. 488, National Engineering Laboratory, Glasgow.

7. S. Ballano, G. Maiseno, P. Mistiando, and F. Wanderlingh, Phys. Rev. 6:1633 (1972).

8. T. Retsina, S. M. Richardson, and W. A. Wakeham, Appl. Sci. Res. 43:325 (1987).

9. N. M. Temme, J. Comput. Phys. 19:324 (1975).

10. S. S. Chen, M. W. Wambsganass, and J. A. Jendrzejczyk, Trans. ASME J. Appl. Mech. 43:325 (1976).

11. L. Bruschi and M. Santini, Rev. Sci. Instrum. 35:1345 (1964).

12. C. M. B. P. Oliveira, Ph.D. thesis (Imperial College, London, 1991).

13. P. S. van der Gulik, R. Mostert, and H. van den Berg, Physica A 151:153 (1988).

14. C. S. Barrett, Structure of Materials (McGraw-Hill, New York, 1952).

15. C. J. Smithells, Metal Reference Book (Butterworths, London, 1949).

16. J. F. Swindells, J. R. Coe, and T. B. Godfrey, J. Res. NBS 48:1 (1952).

17. J. H. Dymond and H. A. Øye, Unpublished result presented to IUPAC Subcommittee on Transport Properties, Thessaloniki, Greece (1990).

18. F. A. Gonçalves, K. Hamano, J. V. Sengers, and J. Kestin, Int. J. Thermophys. 8:641 (1987).

19. B. Knapstad, P. A. Skjolsvik, and H. A. Oye, J. Chem. Eng. Data 34:37 (1989).

20. H. Kashiwagi, T. Hashimoto, Y. Tanaka, H. Kubota, and T. Makita, Int. J. Thermophys. 3:201 (1982).

21. A. K. Doolittle, J. Chem. Eng. Data 9:275 (1964).

22. H. Kashiwagi and T. Makita, Int. J. Thermophys. 3:289 (1982). 